\title{
BMJ Open Risk of dementia in adults with cerebral palsy: a matched cohort study using general practice data
}

\author{
Kimberley J Smith (D , ${ }^{1}$ Mark D Peterson, ${ }^{2}$ Christina Victor, ${ }^{3}$ Jennifer M Ryan ${ }^{4}$
}

To cite: Smith KJ, Peterson MD, Victor C, et al. Risk of dementia in adults with cerebral palsy: a matched cohort study using general practice data. BMJ Open 2021;11:e042652. doi:10.1136/ bmjopen-2020-042652

\section{- Prepublication history and} additional materials for this paper is available online. To view these files, please visit the journal online (http://dx.doi org/10.1136/bmjopen-2020042652).

Received 10 July 2020 Revised 08 November 2020 Accepted 25 November 2020

\section{Check for updates}

\section{Author(s) (or their} employer(s)) 2021. Re-use permitted under CC BY-NC. No commercial re-use. See rights and permissions. Published by BMJ.

${ }^{1} \mathrm{FHMS}$, University of Surrey, Guildford, UK

${ }^{2}$ Physical Medicine \& Rehabilitation, University of Michigan, Ann Arbor, Michigan, USA

${ }^{3}$ College of Medical Health and Life sciences, Brunel University College of Health and Life Sciences, Uxbridge, UK

${ }^{4}$ Public Health and Epidemiology, Royal College of Surgeons in Ireland, Dublin, Ireland

Correspondence to Dr Kimberley J Smith; Kimberley.j.smith@surrey.ac.uk

\section{ABSTRACT}

Objectives Determine the risk of incident dementia in adults with cerebral palsy (CP) compared with age, sex and general practice (GP) matched controls.

Design Retrospective cohort study.

Setting UK GPs linked into the Clinical Practice Research Datalink (CPRD).

Participants CPRD data were used to identify adults aged 18 or older with a diagnosis of CP. Each adult with CP was matched to three controls who were matched for age, sex and GP. In total, 1703 adults with CP and 5109 matched controls were included in the analysis. The mean baseline age of participants was 33.30 years (SD: 15.48 years) and $46.8 \%$ of the sample were female.

Primary outcome New diagnosis of dementia during the follow-up period (earliest date of 1987 to latest date of 2015).

Results During the follow-up, 72 people were identified with a new diagnosis of dementia. The overall proportion of people with and without CP who developed dementia was similar (CP: $n=19,1.1 \%$; matched controls $n=54,10.0 \%$ ). The unadjusted HR suggested that people with $\mathrm{CP}$ had an increased hazard of being diagnosed with dementia when compared with matched controls (HR $2.69,95 \% \mathrm{Cl}$ 1.44 to 5.00). This association was attenuated when CP comorbidities (sensory impairment, intellectual disability and epilepsy) were accounted for (HR 1.92, 95\% Cl 0.92 to 4.02 )

Conclusions There was no difference in the proportion of people with $\mathrm{CP}$ and matched controls who were diagnosed with dementia during the follow-up. Furthermore, while there was evidence for an increased hazard of dementia among people with $\mathrm{CP}$, the fact that this association was attenuated after controlling for comorbidities indicates that this association may be explained by comorbidities rather than being a direct result of CP. Findings should be interpreted with caution due to the low number of incident cases of dementia.

\section{INTRODUCTION}

Cerebral palsy (CP) is the clinical term used for a spectrum of heterogeneous aetiologies and symptoms that result from an injury to the developing human brain. ${ }^{12}$ The most commonly presenting feature of $\mathrm{CP}$ is impaired gross and fine motor functioning, which can lead to difficulties with gait, balance and posture. ${ }^{3}$ However, additional

\section{Strengths and limitations of this study}

This is the first study that has ever looked at the incidence of dementia in adults with cerebral palsy (CP).

- This is a large cohort study with 1703 adults with cerebral palsy (and 5109 age, sex and general practice-matched controls) followed up for a mean of 6.5 years (minimum 0.04 to maximum 28.0 years).

- Only 72 people were diagnosed with dementia during the follow-up, limiting the ability to conduct additional stratified analyses for proposed effect modifiers.

- We were not able to account for subtype of CP or dementia within our analyses.

comorbidities commonly observed in this population can include issues such as intellectual disability (ID), epilepsy, cognitive difficulties, behavioural difficulties and sensory impairments. ${ }^{34}$

Life expectancy for people with CP is similar to the general population, especially in the absence of severe impairments. ${ }^{5} 6$ While the brain injury that causes CP is nonprogressive, ${ }^{7}$ there is evidence that ageing with CP is associated with a higher risk of developing secondary conditions and complications such as frailty, sarcopenia, osteoporosis, osteoarthritis, heart disease and chronic obstructive pulmonary disease. ${ }^{8-11}$ However, there is a lack of research examining whether adults with CP may have an increased risk of developing age-related cognitive disorders, such as dementia.

Dementia is the diagnostic term used to capture a progressive acquired syndrome that impacts brain pathology and presents symptomatically as a substantial decline in cognitive functioning across multiple domains. ${ }^{12}$ It is estimated that 47 million people worldwide live with dementia, and that by 2050 , this will increase to 131 million. $^{13}$ There have been a number of different risk factors proposed to increase a person's risk of developing 
dementia. The most commonly observed in the general population are advanced age, vascular risk factors, poorer baseline cognition, genetics and family history. ${ }^{14}$ However, there is a recognition that there is also an increased risk of dementia in people living with long-term conditions. Common comorbidities in CP such as sensory impairments, ID and epilepsy ${ }^{3}{ }^{4}$ are also known risk factors for the development of dementia. ${ }^{15-17}$ Furthermore, some of the complications that adults with $\mathrm{CP}$ have a higher risk of developing, such as metabolic abnormalities, cardiovascular disease and depression, ${ }^{9} 101819$ are all linked with a greater risk of dementia in the general population..$^{20} 21$

Alongside the possible role of comorbidities and complications in increasing the risk of dementia among people with CP, it has been suggested that CP could lead directly to dementia due to the underlying brain injury. ${ }^{22}$ However, the evidence linking brain injury with dementia is conflicting. ${ }^{23}$

To the best of our knowledge, no peer-reviewed published study has examined whether $\mathrm{CP}$ is associated with dementia. The aim of this study is to examine whether people with $\mathrm{CP}$ have a higher risk of developing dementia than age, sex and general practice (GP)matched controls using longitudinal data from the Clinical Practice Research Datalink (CPRD) database and linked Hospital Episode Statistics (HES).

\section{METHODS \\ Datasets}

We used the CPRD database, which collects consultation data from 4.4 million people in 674 consenting GPs across the UK. ${ }^{24}$ CPRD obtained approval from a national research ethics committee for researchers to use deidentified data for observational research subject to the approval of a study protocol from the Independent Scientific Advisory Committee (online supplemental appendix 1). On approval of a protocol, researchers are able to access deidentified clinical data routinely recorded by GPs such as clinical events, prescriptions, diagnostic testing, lifestyle information, preventative care or anthropometric measures. The data requested for this study covers the period 1987 to 2015 and participants could be enrolled in the study at any time between these years. Participants were followed up for a mean of 6.5 years (minimum 0.04 to maximum 28.0 years).

Diagnosis of dementia in the UK can take place in either a hospital or GP settings. Therefore, we also used patientlevel linked HES data to identify cases of dementia. HES data contains information on hospital utilisation including admissions, outpatient appointments and accident and emergency attendance. CPRD has linked HES data for approximately $60 \%$ of patients in England and Wales.

\section{Participants}

To identify people with CP, we used Read codes (unique alphanumeric codes that link to specific clinical terms relating to CP recorded in GP data, eg, the Read Code F23y400 is linked to the clinical term 'ataxic diplegic CP') (see online supplemental appendix 2). For each patient identified as having CP, we obtained data for three age, sex and GP practice-matched controls without CP. Each included patient was required to be 18 or older and have data that was judged to be research standard (ie, data were of sufficient quality to be used for assessment including criteria such as the patient having a complete and valid first registration date that follows their date of birth and the GP practice not having any significant gaps in recording data).

The index date for the study was the latest of the following: (a1) The date the patient registered with their GP; (2) The date that their data became research standard and (3) The year within which they turned 18. Following exclusions based on these criteria we identified 1705 people with $\mathrm{CP}$ who were matched to 5115 age, sex and GP practice-matched controls without CP. Participants were matched for age based on their year of birth. Two patients with CP and their respective matched controls $(n=8)$ were removed from the analysis as the patients with CP had a diagnosis of dementia identified in HES records that preceded the index date. This left a total of 1703 adults with CP and 5109 matched controls. The index date for each control was set as the same date as their matched patient with CP. For more information about participant selection please see. ${ }^{8918}$

\section{Identification of dementia}

To identify dementia in CPRD, we used Read codes developed by the Cambridge Primary Care $\mathrm{Unit}^{25}$ as well as previous research that has used CPRD data to diagnose dementia. ${ }^{26}$ For a list of read codes used see online supplemental appendix 2. Read codes relating to a history of dementia, family history of dementia or dementia check-up were not included as we were interested in the first recording of a dementia diagnosis. To identify dementia using HES data, we used the following ICD-10 codes: E512, F00, F01, F02, F03, F10.6, F10.7, G30 or G31.0 as documented in previous work. ${ }^{26}$

We identified the date of the first recording of a dementia diagnosis following the index date. For those people who were identified as having a diagnosis of dementia in both the CPRD and HES datasets, we used the earlier date of diagnosis as the event date. Where no event of dementia was identified participants were followed up to the earliest of the following: transfer out of CPRD, death or the end of the follow-up period (November 2015).

\section{Confounders}

We examined chronic conditions associated with both $\mathrm{CP}$ and dementia as potential confounders. Using Read Codes in Smith et $a l^{18}$ Ryan et at or the Cambridge Primary Care Unit codelists ${ }^{25}$ we identified diabetes, heart disease, stroke, depression, ID, epilepsy and sensory impairment (visual and/or hearing impairment). For each condition, we only included those that occurred before the dementia 
event date (eg, a depression diagnosis needed to be made prior to the dementia diagnosis to be included as a confounder). We also included average annual GP visits as a potential confounder, as people with CP may attend their GP more frequently than those without $\mathrm{CP}$, and those who attend the GP more often may be more likely to be diagnosed with dementia. We categorised average GP visits as 0-2 visits per year, 2-11.9 visits per year or $\geq 12$ visits per year following Smith $e t a l^{18}$

\section{Statistical analysis}

Descriptive statistics were calculated to present the characteristics of the sample, and cross-tabulations calculated to determine potential differences in baseline characteristics between people with CP and matched controls. A descriptive analysis was performed to determine the ages at which dementia diagnoses were made in people with $\mathrm{CP}$ and matched controls. We also calculated the incidence rate of dementia per 1000 person-years for both groups.

We used stratified Cox proportional hazards regression with dementia diagnosis as the outcome to compare the hazard of dementia between patients with and without CP. The hazards regression was first run unadjusted (model 1). In model 2, we adjusted for CP comorbidities that have been proposed to be risk factors for dementia (ID, sensory impairment and epilepsy). In model 3, we adjusted for CP complications also associated with dementia risk (diabetes, stroke, heart disease) and average GP visits.

Prior to running the Cox proportional hazards regression, we plotted scaled Schoenfeld residuals against time to assess the assumption of proportional hazards, and the assumption of proportionality was met. All analyses were conducted using STATA V.16.0.

\section{Sensitivity analyses}

We reran the model excluding any cases of dementia that were identified within 12 months of the index date, as these could indicate prevalent rather than incident cases of dementia (as on registering at a new practice a person may have any prevalent conditions recorded by their new GP as a diagnosis). We also ran a second sensitivity analysis only including people aged 40 or older at baseline to account for the fact that dementia is typically seen in people who are older.

\section{Patient and public involvement}

There was no patient and public involvement in the design of this study.

\section{RESULTS}

\section{Descriptive data: participant characteristics}

The mean age of the sample (both adults with $\mathrm{CP}$ and their matched controls) was 33.3 years (SD: 15.5 years) and $46.8 \%(\mathrm{n}=3118)$ of the sample were female. Sample characteristics stratified by CP status are described in table 1. Cross-tabulation analyses revealed that the sample with $\mathrm{CP}$ were more likely to have a higher annual number of GP visits, and more likely to have a baseline diagnosis of stroke, epilepsy and/or sensory impairment (table 1). In addition, we found that the sample with $\mathrm{CP}$ were less likely to have a baseline diagnosis of diabetes or heart disease than the matched control group (table 1).

\section{Risk of dementia}

In total, 72 people were diagnosed with dementia during the follow-up period (table 2). A total of $53(1.04 \%$ ) people from the matched control group developed dementia over a median of 10.95 years of follow-up (minimum of 0.14 years to maximum of 28.01 years). Whereas a total of $19(1.12 \%)$ people with CP developed dementia over a median of 7.15 years of follow-up (minimum of 0.04 years to maximum of 27.94 years). The dementia incidence rate for people with CP was 0.0013 per 1000 person-years, and 0.0009 per 1000 person-years (table 2).

Unadjusted stratified Cox modelling indicated that people with $\mathrm{CP}$ had an increased hazard of dementia compared with age-matched, sex-matched and GP-matched controls without CP (HR 2.69, 95\% CI 1.44 to 5.00, $\mathrm{p}=0.002$ ). However, after adjusting for $\mathrm{CP}$ comorbidities the association became non-significant (HR $1.92,95 \% \mathrm{CI} 0.92$ to $4.02, \mathrm{p}=0.08$ ) and remained nonsignificant in the fully-adjusted model (see table 2). Of the 72 people who were diagnosed with dementia we found that $47 \%(n=9)$ of people with CP who were diagnosed with dementia were aged 65 years or younger, compared with 5.7\% ( $\mathrm{n}=3$ ) of people who did not have CP (table 3).

Results from our first sensitivity analysis excluding any dementia diagnoses made within 12 months of the index date revealed that a total of 51 people without CP $(1.00 \%)$ and 16 people with CP $(0.94 \%)$ were diagnosed with dementia (online supplemental appendix 3). As with the primary analysis, there was evidence for an unadjusted increased hazard of dementia in adults with CP when compared with matched controls (HR 2.50, $95 \%$ CI 1.28 to $4.86, p=0.007$ ), whereas after adjusting for CP comorbidities, the association was attenuated (HR $1.95,95 \%$ CI 0.90 to $4.22, \mathrm{p}=0.09$ ) and remained non-significant in the fully adjusted model (HR 1.90, $95 \%$ CI 0.75 to $4.79, \mathrm{p}=0.18$ ). For our second sensitivity analysis, we examined whether only including people aged 40 or older at baseline had an impact on our results (see online supplemental appendix 3). For this analysis, the sample size was reduced to 490 people with CP and 1470 matched controls. A total of $16(3.27 \%)$ of people with CP developed dementia, whereas $52(3.54 \%)$ of the matched controls developed dementia. The results from the Cox proportional hazards regression also indicated an increased hazards of developing dementia in adults with CP when compared with matched controls in unadjusted analyses (HR 2.46, 95\% CI 1.20 to 4.33, $\mathrm{p}=0.014$ ), which was also attenuated after accounting for $\mathrm{CP}$ comorbidities (HR $1.07,95 \%$ CI 0.70 to $3.36, \mathrm{p}=0.29$ ) (see online supplemental appendix 3). 
Table 1 Characteristics of participants

\begin{tabular}{|c|c|c|c|c|c|c|}
\hline & \multicolumn{2}{|c|}{ Cerebral palsy $(n=1703)$} & \multicolumn{2}{|c|}{ Matched controls $(n=5109)$} & \multirow[b]{2}{*}{$\mathrm{X}^{2}$} \\
\hline & & $\mathbf{N}$ & $\%$ & $\mathbf{N}$ & $\%$ & \\
\hline \multirow[t]{5}{*}{ Baseline age } & $<40$ & 1213 & 71.22 & 3639 & 71.23 & - \\
\hline & $40-49$ & 223 & 13.68 & 669 & 13.09 & \\
\hline & $50-59$ & 134 & 7.87 & 402 & 7.87 & \\
\hline & $60-69$ & 77 & 4.52 & 231 & 4.52 & \\
\hline & $\geq 70$ & 56 & 3.29 & 168 & 3.29 & \\
\hline \multirow[t]{2}{*}{ Sex } & Male & 906 & 53.20 & 2718 & 53.20 & - \\
\hline & Female & 797 & 46.80 & 2391 & 46.80 & \\
\hline \multirow[t]{3}{*}{ Average GP visits per year } & $0-2$ per year & 133 & 7.80 & 716 & 14.01 & $X^{2}=329.14, p<0.001$ \\
\hline & 2.1-11.9 per year & 1177 & 69.11 & 4029 & 78.86 & \\
\hline & $\geq 12$ per year & 393 & 23.08 & 364 & 7.12 & \\
\hline \multirow[t]{2}{*}{ Depression } & Yes & 310 & 18.20 & 864 & 16.91 & $X^{2}=1.494, p=0.22$ \\
\hline & No & 1393 & 81.80 & 4245 & 83.09 & \\
\hline \multirow[t]{2}{*}{ Diabetes } & Yes & 60 & 3.52 & 253 & 4.95 & $X^{2}=5.95, p=0.02$ \\
\hline & No & 1643 & 96.48 & 4856 & 95.05 & \\
\hline \multirow[t]{2}{*}{ Heart disease } & Yes & 182 & 10.69 & 640 & 12.53 & $X^{2}=4.08, p=0.04$ \\
\hline & No & 1521 & 89.31 & 4469 & 87.47 & \\
\hline \multirow[t]{2}{*}{ Stroke } & Yes & 69 & 4.05 & 99 & 1.94 & $X^{2}=23.73, p<0.001$ \\
\hline & No & 1634 & 95.95 & 5010 & 98.06 & \\
\hline \multirow[t]{2}{*}{ Sensory impairment } & Yes & 298 & 17.50 & 535 & 10.47 & $X^{2}=58.76, p<0.001$ \\
\hline & No & 1405 & 82.50 & 4574 & 89.53 & \\
\hline \multirow[t]{2}{*}{ Epilepsy } & Yes & 427 & 25.07 & 70 & 1.37 & $X^{2}=928.36, p<0.001$ \\
\hline & No & 1276 & 74.93 & 5039 & 98.63 & \\
\hline \multirow[t]{2}{*}{ Intellectual disability } & Yes & 361 & 21.20 & 24 & 0.47 & $X^{2}=1000, p<0.001$ \\
\hline & No & 1342 & 78.80 & 5085 & 99.53 & \\
\hline
\end{tabular}

This table presents the distribution of the sociodemographic and health-related characteristics of patients within the sample.

\section{DISCUSSION}

Results from this analysis provide the first evidence that $\mathrm{CP}$ is associated with an increased hazard of developing dementia, but that this increased hazard is explained by the presence of $\mathrm{CP}$ comorbidities. It is also worth noting that the proportion of those who developed dementia did not differ between people with and without $\mathrm{CP}$ and the overall incidence rate of dementia was low for both groups. However, a higher proportion of those people with $\mathrm{CP}$ who were diagnosed with dementia were diagnosed with dementia at 65 or younger when compared with those who did not have CP though the low number of dementia cases and descriptive analyses limit inferences.

Our results indicated that the observed association between CP and dementia was attenuated after adjusting for CP comorbidities. However, there is heterogeneity in

Table 2 Risk of dementia in people with CP $(n=1703)$ compared with age, sex and GP practice-matched controls $(n=5109)$

\begin{tabular}{|c|c|c|c|c|c|c|c|}
\hline & Events $\mathrm{n}$ & $\begin{array}{l}\text { Events } \\
\%\end{array}$ & $\begin{array}{l}\text { Person- } \\
\text { years in } \\
1000 \text { s }\end{array}$ & $\begin{array}{l}\text { Incidence per } 1000 \\
\text { person-years }(95 \% \mathrm{Cl})\end{array}$ & $\begin{array}{l}\text { Model } 1 \mathrm{HR} \\
(95 \% \mathrm{Cl}) \text { and } \mathrm{p} \\
\text { value }\end{array}$ & $\begin{array}{l}\text { Model } 2 \mathrm{HR} \\
(95 \% \mathrm{Cl}) \text { and } \mathrm{p} \\
\text { value }\end{array}$ & $\begin{array}{l}\text { Model } 3 \mathrm{HR} \\
(95 \% \mathrm{Cl}) \text { and } \mathrm{p} \\
\text { value }\end{array}$ \\
\hline No CP & 53 & 1.04 & 56.66 & 0.00094 (0.0007 to 0.0012$)$ & 1 (reference) & 1 (reference) & 1 (reference) \\
\hline $\mathrm{CP}$ & 19 & 1.12 & 14.57 & 0.00130 (0.0008 to 0.0020$)$ & $\begin{array}{l}2.69(1.44 \text { to } 5.00), \\
p=0.002\end{array}$ & $\begin{array}{l}1.92(0.92 \text { to } \\
4.02), p=0.08\end{array}$ & $\begin{array}{l}1.76 \text { ( } 0.73 \text { to } \\
4.25), p=0.21\end{array}$ \\
\hline
\end{tabular}

Model 1: unadjusted.

Model 2: adjusted for baseline (ie, predementia) ID, sensory impairments and epilepsy.

Model 3: adjusted for model 2 plus baseline (ie, predementia) diagnosis of diabetes, heart disease, stroke, depression and average annual GP visits.

CP, cerebral palsy; GP, general practice; ID, intellectual disability. 
Table 3 Age at dementia diagnosis

\begin{tabular}{llcccc}
\hline \multirow{2}{*}{$\begin{array}{l}\text { Age at } \\
\text { diagnosis }\end{array}$} & \multicolumn{2}{l}{$\begin{array}{l}\text { CP diagnosed with } \\
\text { dementia }(\mathbf{n = 1 9 )}\end{array}$} & & \multicolumn{2}{l}{$\begin{array}{l}\text { No CP diagnosed } \\
\text { with dementia }(\mathbf{n}=53)\end{array}$} \\
\cline { 2 - 3 } \cline { 5 - 6 } & $\mathbf{N}$ & $\%$ & & $\mathbf{N}$ & $\%$ \\
\hline$<50$ & 3 & 15.79 & & 2 & 3.77 \\
$50-65$ & 6 & 31.58 & & 2 & 3.77 \\
$66-80$ & 3 & 15.79 & & 17 & 32.08 \\
$\geq 80$ & 7 & 36.84 & & 32 & 60.38 \\
\hline
\end{tabular}

$\mathrm{CP}$, cerebral palsy.

the kinds of comorbidities that people with CP can present with in adulthood. Brown and Eunson ${ }^{27}$ suggested that comorbidities in CP can be split into cocausal comorbidities (comorbidities caused by the same underlying brain pathology which includes ID), complications (secondary comorbidities that arise due to complications of living with CP, such as osteoarthritis) and co-occurring comorbidities (comorbidities that are not linked with CP directly). There is evidence that cocausal, co-occurring comorbidities and complications linked with CP are all linked with an increased risk of dementia in general population samples. Cocausal and co-occurring comorbidities such as ID, epilepsy and sensory impairments are all independently associated with an increased risk of dementia. ${ }^{15-17}$ Furthermore, complications such as cardiovascular disease and depression are also linked with an increased risk of dementia. ${ }^{20} 21$

The results from our analyses indicated that the increased hazard was attenuated when we controlled for cocausal CP comorbidities: sensory impairment, epilepsy and ID. However, it is worth noting that while all these CP comorbidities have been linked to dementia, ${ }^{15-17}$ it is ID that is most consistently implicated as being a risk factor for dementia. ${ }^{28} \mathrm{It}$ is estimated that $20 \%-45 \%$ of people with CP have comorbid ID, ${ }^{29} 30$ and evidence from the general population indicates that ID is linked with a greater risk of dementia. ${ }^{15}$ In people with CP the presence of ID may be linked to increased grey matter pathology, ${ }^{30}$ which is linked with an increased risk of developing dementia. ${ }^{31}$ It is possible that comorbid ID could be an important modifier of dementia risk in this population. However, due to the low number of participants who developed dementia we were unable to conduct stratified analyses to examine this with our data.

Due to the low number of people with CP who developed dementia, we were not able to look at which risk factors were linked with an increased risk of dementia in adults with CP. There are a broad range of risk factors implicated in dementia risk beyond CP comorbidities such as vascular risk factors, older age, baseline cognition, lifestyle and genetic risk. ${ }^{14}$ Future work could examine the risk factors that predict dementia in people living with CP and whether these are the same or different to the risk factors observed in the general population. If risk factors in adults with $\mathrm{CP}$ are different to the general population, this would indicate that targeted interventions and screening for adults with CP would be important.
In thinking about CP and risk of dementia, we also need to be aware of the contemporary landscape of dementia research. There is an increasing awareness that rather than focusing on individual risk factors that we need to examine clusters of risk factors. A systematic review published in 2019 indicated that as the number of risk factors associated with dementia increased that the risk of developing dementia also increased. ${ }^{32}$ Interestingly people with ID have a higher likelihood of having additional CP comorbidities that also increase the risk of dementia such as epilepsy ${ }^{30}$ and sensory impairment. ${ }^{34}$ Therefore, future work should consider how $\mathrm{CP}$ comorbidities could cluster together in predicting the risk of dementia in adults with CP.

While this is the first study that examined the risk of dementia in people with CP, there are a number of limitations that should be borne in mind when interpreting results. Due to the small number of people who developed dementia over the follow-up generalisability of the results may be limited. Furthermore, while there were some interesting observations within this study (such as the higher proportion of people with CP who were diagnosed with an early-onset dementia which is defined as onset at 65 or younger), the low numbers of participants who were diagnosed with dementia meant that we were not able to explore this finding with formal inferential statistics. An additional limitation pertains to the dataset used; there is no study that has formally examined the sensitivity of CPRD data for identifying people with CP which could lead to possible issues with missing data for adults with CP. It is also worth noting that HES data only captured $60 \%$ of practice in England and Wales, so there is also a possibility of missing dementia diagnoses. There is a need for more work (with larger cohorts) to determine whether CP is linked with an increased risk of early-onset dementia, and whether this is explained by CP comorbidities.

It is also worth noting that CP is the umbrella diagnosis given to a heterogeneous spectrum of aetiologies and physical symptoms. We were unable to account for this heterogeneity within this study (eg, CP subtype, presence and severity of brain pathology, baseline cognitive functioning or gross motor function). More careful consideration of CP heterogeneity could reveal interesting insights into the mechanisms explaining the observed findings. Finally, there are different subtypes of dementia (eg, Vascular dementia, Alzheimer's disease, etc), and we did not explore associations with each type within our study.

Our results indicate a clear need for future research studies to examine the risk of dementia associated with CP in order to better understand whether CP is linked with dementia, and whether this risk might be explained by CP comorbidities as suggested in this study. There is also a broader need for more work to examine cognitive decline and dementia in this population, and work to help us understand what factors could predict cognitive decline and dementia in order to target interventions. This could have important clinical applications, as there is currently little guidance around clinical monitoring for cognitive decline or dementia in adults with C.P.

To conclude, this research provides the first evidence that adults with CP may have an increased hazard of being 
diagnosed with dementia compared with the general adult population but that this is likely driven by CP comorbidities rather than being a direct impact of having a diagnosis of CP. More research is needed to confirm this finding and determine which specific comorbidities may drive the association between $\mathrm{CP}$ and dementia.

Acknowledgements Additional contributors to the overall project and protocol who were not involved in this particular substudy were Nana Anokye (Brunel University London), Neil O'Connell (Brunel University London), Nicola Ryan (Hospital Clínico San Carlos) and Silvia Liverani (Queen Mary's University London).

Contributors JR was the lead for the project and protocol submitted to CPRD (see online supplemental appendix 2), and all authors (KS, JR, CV and MDP) were involved in the design of the study. JR and KS conducted the analyses for this study. KS drafted the manuscript and all study authors had input into revising the manuscript (KS, JR, CV and MDP).

Funding This study was supported by a Research Catalyst Award from Brunel University London.

Competing interests None declared.

Patient consent for publication Not required.

Provenance and peer review Not commissioned; externally peer reviewed.

Data availability statement Data may be obtained from a third party and are not publicly available. Access to CPRD data is only given to named researchers on approval of a protocol, and researchers agree to a data sharing agreement that only allows data to be accessed by named researchers. The data are not openly accessible and it is not possible to share data. We have shared the readcodes used for this analysis in online supplemental appendix 2.

Supplemental material This content has been supplied by the author(s). It has not been vetted by BMJ Publishing Group Limited (BMJ) and may not have been peer-reviewed. Any opinions or recommendations discussed are solely those of the author(s) and are not endorsed by BMJ. BMJ disclaims all liability and responsibility arising from any reliance placed on the content. Where the content includes any translated material, BMJ does not warrant the accuracy and reliability of the translations (including but not limited to local regulations, clinical guidelines, terminology, drug names and drug dosages), and is not responsible for any error and/or omissions arising from translation and adaptation or otherwise.

Open access This is an open access article distributed in accordance with the Creative Commons Attribution Non Commercial (CC BY-NC 4.0) license, which permits others to distribute, remix, adapt, build upon this work non-commercially, and license their derivative works on different terms, provided the original work is properly cited, appropriate credit is given, any changes made indicated, and the use is non-commercial. See: http://creativecommons.org/licenses/by-nc/4.0/.

\section{ORCID iD}

Kimberley J Smith http://orcid.org/0000-0002-1323-627X

\section{REFERENCES}

1 Rosenbaum P, Paneth N, Leviton A, et al. A report: the definition and classification of cerebral palsy April 2006. Dev Med Child Neurol Suppl 2007;109:8-14.

2 Blair E, Watson L. Epidemiology of cerebral palsy. In: Seminars in fetal and neonatal medicine. . Elsevier, 2006: 11. 117-25.

3 Richards CL, Malouin F. Cerebral palsy: definition, assessment and rehabilitation. In: Handbook of clinical neurology. Elsevier, 2013: 183-95.

4 Sankar C, Mundkur N. Cerebral palsy-definition, classification, etiology and early diagnosis. Indian J Pediatr 2005;72:865-8.

5 Blair E, Langdon K, Mclntyre S, et al. Survival and mortality in cerebral palsy: observations to the sixth decade from a data linkage study of a total population register and national death index. BMC Neurol 2019;19:111.

6 Colver A. Outcomes for people with cerebral palsy: life expectancy and quality of life. Paediatr Child Health 2012;22:384-7.

7 Bromham N, Dworzynski K, Eunson P, et al. Cerebral palsy in adults: summary of NICE guidance. BMJ 2019;364:I806.
8 O'Connell NE, Smith KJ, Peterson MD, et al. Incidence of osteoarthritis, osteoporosis and inflammatory musculoskeletal diseases in adults with cerebral palsy: a population-based cohort study. Bone 2019;125:30-5.

9 Ryan JM, Peterson MD, Matthews A, et al. Noncommunicable disease among adults with cerebral palsy: a matched cohort study. Neurology 2019;93:e1385-96.

10 Ryan JM, Allen E, Gormley J, et al. The risk, burden, and management of non-communicable diseases in cerebral palsy: a scoping review. Dev Med Child Neurol 2018;60:753-64.

11 Whitney DG, Hurvitz EA, Devlin MJ, et al. Age trajectories of musculoskeletal morbidities in adults with cerebral palsy. Bone 2018;114:285-91.

12 Arvanitakis Z, Shah RC, Bennett DA. Diagnosis and management of dementia: review. JAMA 2019;322:1589-99.

13 Alzheimer's Disease International. World Alzheimer report 2015. the global impact of dementia: an analysis of prevalence, incidence, cost and trends. London: Alzheimer's Disease International, 2015.

14 Hou X-H, Feng L, Zhang C, et al. Models for predicting risk of dementia: a systematic review. J Neurol Neurosurg Psychiatry 2019;90:373-9.

15 Strydom A, Chan T, King M, et al. Incidence of dementia in older adults with intellectual disabilities. Res Dev Disabil 2013;34:1881-5.

16 Panza F, Solfrizzi V, Logroscino G. Age-Related hearing impairment-a risk factor and frailty marker for dementia and AD. Nat Rev Neurol 2015;11:166-75.

17 Coulehan K, Bender HA. Evaluation of Comorbid Epilepsy and Dementia. In: Handbook on the neuropsychology of aging and dementia. Springer, 2019: 641-60.

18 Smith KJ, Peterson MD, O'Connell NE, et al. Risk of depression and anxiety in adults with cerebral palsy. JAMA Neurol 2019;76:294-300.

19 McPhee PG, MacDonald MJ, Cheng JL, et al. Emerging evidence for accelerated ageing and cardiovascular disease in individuals with cerebral palsy. J Rehabil Med 2019;51:525-31.

20 Whitmer RA, Sidney S, Selby J, et al. Midlife cardiovascular risk factors and risk of dementia in late life. Neurology 2005;64:277-81.

21 Diniz BS, Butters MA, Albert SM, et al. Late-life depression and risk of vascular dementia and Alzheimer's disease: systematic review and meta-analysis of community-based cohort studies. Br J Psychiatry 2013;202:329-35.

22 O'Brien G, Bass A, Rosenbloom L. Chapter 4: Cerebral palsy and ageing. In: O'Brien G, Rosenbloom L, eds. Developmental disability and ageing, 2009: 39-52.

23 Godbolt AK, Cancelliere C, Hincapié CA, et al. Systematic review of the risk of dementia and chronic cognitive impairment after mild traumatic brain injury: results of the International collaboration on mild traumatic brain injury prognosis. Arch Phys Med Rehabil 2014;95:S245-56.

24 Herrett E, Gallagher AM, Bhaskaran K, et al. Data resource profile: clinical practice research Datalink (CPRD). Int J Epidemiol 2015;44:827-36

25 Unit, C.P.C. CPRD @ Cambridge - code lists. Available: http://www. phpc.cam.ac.uk/pcu/cprd_cam/codelists/ [Accessed Mar 2018].

26 Brown A, Kirichek O, Balkwill A, et al. Comparison of dementia recorded in routinely collected hospital admission data in England with dementia recorded in primary care. Emerg Themes Epidemiol 2016;13:11

27 Brown JK. Euson, Heterogeneity in cerebral palsy: variations in neurology, comorbidity and associated conditions. In: Bax M, Gillberg C, eds. Comorbidities in developmental disorders. London: MacKeith Press, 2010.

28 Sheehan R, Ali A, Hassiotis A. Dementia in intellectual disability. Curr Opin Psychiatry 2014;27:143-8.

29 Kancherla V, Amendah DD, Grosse SD, et al. Medical expenditures attributable to cerebral palsy and intellectual disability among Medicaid-enrolled children. Res Dev Disabil 2012;33:832-40.

30 Reid SM, Meehan EM, Arnup SJ, et al. Intellectual disability in cerebral palsy: a population-based retrospective study. Dev Med Child Neurol 2018;60:687-94.

31 Ikram MA, Vrooman HA, Vernooij MW, et al. Brain tissue volumes in relation to cognitive function and risk of dementia. Neurobiol Aging 2010;31:378-86.

32 Peters R, Booth A, Rockwood K, et al. Combining modifiable risk factors and risk of dementia: a systematic review and meta-analysis. BMJ Open 2019;9:e022846.

33 Robertson J, Hatton C, Emerson E, et al. Prevalence of epilepsy among people with intellectual disabilities: a systematic review. Seizure 2015;29:46-62.

34 Carvill S. Sensory impairments, intellectual disability and psychiatry. J Intellect Disabil Res 2001;45:467-83. 